\section{Dental HIV transmission?}

SIR - It is frequently reported, on the basis of the conclusion reached by $\mathrm{Ou} e t$ $a l .{ }^{1}$, that a Florida dentist infected five of his eight HIV-1 seropositive patients. The molecular data, consisting of nucleotide sequences from the highly variable $\mathrm{C} 2-\mathrm{V} 3$ region of the $\mathrm{HIV}-1$ envelope gene, are particularly crucial because the epidemiological investigation was retrospective: all the patients were aware of the dental transmission hypothesis ${ }^{2}$. Because this case remains unique in terms of the hypothesized

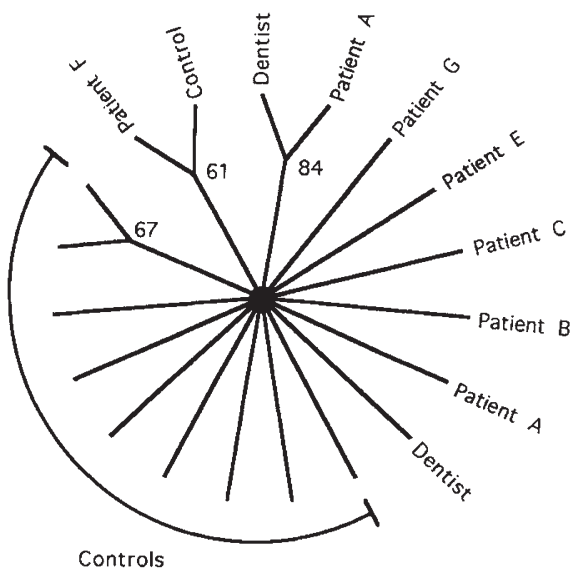

Statistical analysis of $\mathrm{C} 2-\mathrm{V} 3$ sequence data: 100 bootstrap replicates were analysed by threshold parsimony (threshold value 2.0). Very few nodes were found in more than $50 \%$ of the replicates, indicating that there is little reliable phylogenetic information in these data (no inference about relationships should be drawn from the relative positions of branches coming from the central node). The 'dental group' consists of patients $A$, $B, C, E$ and $G$. Additional details can be obtained from R. W. D.

mode of virus transmission, the conclusion and analyses used to support it should be carefully examined.

What is the null hypothesis against which the dental transmission hypothesis should be tested? Population genetics suggests that a rapidly evolving marker can develop strong geographical substructure; therefore, an appropriate null hypothesis is that the patients independently acquired similar variants within the local community. These two hypotheses can be distinguished by the inferred historical relationships among the viral sequences. The dental transmission hypothesis requires that a branch on the viral phylogenetic tree lead to the dental group alone and not include any controls. In phylogenetic terms, the dental group must be monophyletic. The null hypothesis would be rejected if a tree with a monophyletic dental group is significantly better supported than any tree with controls intermixed within the dental group.

NATURE · VOL 361 - 25 FEBRUARY 1993
We tested these hypotheses using new sequences from the dental patients and a new set of regional controls. Ou et al. selected four controls that were similar to the dentist's sequences for detailed analysis, and we selected ten controls. This selection is justified: the dental group should be monophyletic compared to any controls. In fact, the test is biased in favour of accepting the dental transmission hypothesis because the controls in both studies were obtained at clinics about 90 miles from the dentist's practice area.

Although parsimony analysis gives a monophyletic dental group, some trees that mix controls and the dental group require only one additional substitution. One weakness of parsimony analysis is the assumption that all nucleotide changes are approximately equally likely. This is unlikely to be the case for the $\mathrm{C} 2-\mathrm{V} 3$ region; many sites are identical across North America, whereas other sites commonly vary within individuals. A slightly different analysis, threshold parsimony, allows for such a situation $^{3}$. All the best threshold parsimony trees include one or more controls within the dental group, whether our data or those of Ou et al. are used.

Parsimony analysis yields a point estimate, whereas the hypothesis test requires an estimate of confidence intervals. Ou et al. used bootstrap resampling and reported finding a monophyletic dental group in $79 \%$ of the replicates. In contrast, we find a monophyletic dental group in only $59 \%$ of the replicates, and in fewer than $50 \%$ of the replicates using threshold parsimony, a result due to the greater similarity of our controls to the dental group (see figure). The exact level required for significance in the bootstrap test is not known in general, but in the simplest case (four sequences and a molecular clock) greater than $80 \%$ is required, and values less than $75 \%$ mean that the relationship in question cannot be resolved ${ }^{4}$.

Ou et al. used two additional methods, but neither provides a direct test of the dental transmission hypothesis. The Wilcoxon rank-sum test is inappropriate because it compares average properties, not individual properties, of the patient and control groups. The other test used a pattern of eight noncontiguous amino acids said to form the dentist's 'signature'. In fact, none of the signature residues is unique to the dental group; one of our controls has six of the eight, and five have four or more. Further, examining the signature as phylogenetic data reveals that seven of the sites provide evidence for groups containing both patients and controls.
Our analyses show that the available data are consistent with both the dental transmission hypothesis and the null hypothesis and do not yet distinguish between the two. Much of the difficulty lies in the decision of $\mathrm{Ou}$ et al. to focus on the small, highly variable $\mathrm{C} 2-\mathrm{V} 3$ region. A more conclusive test of the hypothesis that these patients were infected with HIV during dental treatment will require a larger dataset from some other region of the HIV genome.

\section{Ronald W. DeBry}

\section{Lawrence G. Abele}

Department of Biological Science,

Florida State University,

Tallahassee,

Florida 32306, USA

Stanley H. Welss

Division of Infectious Diseases

Epidemiology,

Department of Preventive Medicine and Community Health,

New Jersey Medical School,

Newark,

New Jersey 07107, USA

Martin D. HIII

Maria Bouzas

Eric Lorenzo

Folke Graebnitz

Llonel Resnick

Retrovirology Research Laboratories,

Mount Sinai Medical Center,

Miami Beach,

Florida 33140, USA

1. Ou, C.-Y. et al. Science 256, 1165-1171 (1992)

2. Ciesielski, C. et al. Ann. intern. Med. 116, 798-805 (1992).

3. Felsenstein, J. Biol. J. Linnean Soc. 16, 183-196 (1981).

4. Zharkikh, A. \& Li, W.-H. Molec. Biol. Evol. 9, 1119 1147 (1992).

\section{Rose is a rose}

SIR - I apologize to your readers for any confusion caused by my deducing the names of genes from those of mutants ${ }^{1}$, thank Enoch et al. for correcting $\mathrm{me}^{2}$, and blame Gertrude Stein for suggesting the idea that a mutant names a gene names a protein ${ }^{3}$. Finally, in an effort to minimize confusion about the hus mutants, I note that their names appear to have changed between Enoch et al.'s first ${ }^{4}$ and second ${ }^{5}$ publications on the subject (for example hus14 in ref. 4 became hus1-14 in ref. 5).

\section{Andrew Murray}

Department of Physiology,

University of California,

San Francisco,

California 94143-0444, USA

\footnotetext{
1. Murray, A. W. Nature 359, 599-604 (1992).

. Enoch, T., Carr, A. \& Nurse, P. Nature 361, 26 (1993)

3. Stein, G. Sacred Emily (Something Else, New York 1968)

4. Enoch, T., Gould, K. L. \& Nurse, P. Cold Spring Harb. Symp. quant. Biol. 56, 409-416 (1991)

5. Enoch, T., Carr, A. M. \& Nurse, P. Genes Dev. 6 2035-2046 (1992)
} 